\title{
Antes que la naturaleza muera: de la Primavera silenciosa a Nuestro futuro robado
}

\author{
Before nature dies: from silent spring to our stolen future
}

(D) Víctor Pulido Capurro ${ }^{1}$, (D) Augusto Dalmau García Bedoya ${ }^{2}$ y

(iD) Edith Olivera Carhuaz ${ }^{3}$

\footnotetext{
${ }^{1}$ Universidad Privada San Juan Bautista, Lima, Perú

2.Universidad Le Cordon Bleu. Lima, Perú

3.Universidad Inca Garcilaso de la Vega. Lima, Perú
}

Recibido: 16/04/2021 Revisado: 24/05/2021 Aceptado: 16/06/2021 Publicado: 31/06/2021

\section{RESUMEN}

Se presenta los aspectos más resaltantes de la publicación de tres libros, la Primavera silenciosa por Rachel Louise Carson, publicado en 1962; Antes que la Naturaleza muera escrito por Jean Dorst, publicado en 1965, y Nuestro futuro robado escrito por Theo Colborn, Dianne Dumanoski y John Peterson Myers, publicado en 1996, los cuales basados en evidencias científicas causaron un gran impacto en la población mundial, debido a que llamaron la atención acerca de la destrucción de la naturaleza, la pérdida de especies de animales y plantas, del uso discriminado de pesticidas como el DDT y de sustancias químicas que se comportan como disruptores que alteran el sistema endocrino de los organismos. Ponen en evidencia la importancia de los temas sociales y ecológicos y reconoce la necesidad de una reflexión desde la bioética y la educación ambiental, que forman parte del mismo discurso que relaciona lo científico y lo humano. Y de cómo estos libros de algún modo han influenciado en una serie de procesos y mecanismos y acuerdos que se han concretado para proteger al planeta, los hábitats y las especies. Palabras clave: Antes que la naturaleza muera, primavera silenciosa, nuestro futuro robado, ambiente, pesticidas, disruptores.

\section{ABSTRACT}

The most outstanding aspects of the following three books are presented, the Silent Spring by Rachel Louise Carson, published in 1962; Before Nature Dies written by Jean Dorst, published in 1965, and Our Stolen Future written by Theo Colborn, Dianne Dumanoski and John Peterson Myers, published in 1996, which are based on scientific evidence, caused a great impact on the world population, due to which they called attention to these issues; destruction of nature, the loss of species of animals and plants, the discriminatory use of pesticides such as DDT and chemicals that behave as disruptors that alter the endocrine system of organisms. They highlight the importance of social and ecological issues and recognize the need for a reflection from bioethics and environmental education, which are part of the same speech that relates the scientific and the human. Also, how these books have somehow influenced a series of processes 
Antes que la naturaleza muera: de la Primavera

Pulido, V. y cols.

silenciosa a Nuestro futuro robado

and mechanisms and agreements that have been made to protect the planet, habitats and species. Keywords: Before nature dies, silent spring, our stolen future, environment, pesticides, disruptors.

\section{INTRODUCCIÓN}

Tres grandes obras impactaron en el siglo XX sobre temas ambientales, la Primavera silenciosa escrito por Rachel Louise Carson en 1962; Antes que la Naturaleza Muera de Jean Dorst en 1972 y Nuestro futuro robado por Theo Colborn, Dianne Dumanoski y John Peterson Myers en 1996, los cuales se han convertido en clásicos de la literatura ecológica, y por tanto inspiraron a numerosas personas e instituciones, a levantar su voz de protesta a favor de la defensa de la conservación de la naturaleza.

La aparición de estas importantes tres obras, en el periodo de 34 años, les ha permitido transitar por la misma línea de pensamiento. La Primavera silenciosa centra su atención en el uso indiscriminado de los plaguicidas, especialmente cuando a partir de los años 1959 se encuentran evidencias de la acción del DDT en la cadena alimenticia y su acumulación en el tejido adiposo de los animales; Antes que la naturaleza muera narra la destrucción de la naturaleza por efecto de la acción devastadora del hombre, y Nuestro futuro robado alerta sobre la disrupción endocrina, que pone en evidencia el riesgo del uso de las sustancias químicas, las cuales pueden interferir en las hormonas y trastornan los procesos de reproducción y desarrollo del ser humano, poniendo en serio peligro el futuro de la especie.

Pero ¿cuáles fueron los impactos que produjeron estas obras con su publicación? Los impactos fueron muchos. Uno de los más importantes ha sido poner en evidencia ante la opinión pública lo dañino que significa para los animales y la salud humana el uso de pesticidas y determinado tipo de sustancias quí- micas; seguidamente la conciencia pública se incrementó en defensa de la naturaleza y asumió una posición más proactiva a favor de la conservación del ambiente, las plantas, los animales y las personas; también generó el inicio de una serie de reuniones de carácter internacional, en que tanto los gobiernos como la sociedad civil, asumieron una posición más activa a favor de la conservación del ambiente.

Por lo tanto, el presente artículo constituye una reflexión acerca del significado de estos tres libros en el desarrollo de la conciencia ambientalista mundial, así como para tener mayor cuidado en el uso de sustancias tóxicas a fin de garantizar un mundo mejor para todos los seres vivos que habitamos este planeta.

\section{Antes que la naturaleza muera}

Jean Dorst, biólogo y naturalista francés publicó en 1972, uno de los libros más importantes de la literatura ambientalista cuyo título original en francés es Avant que nature meu$\boldsymbol{r}$. Durante el tiempo que escribió esta monumental obra, el famoso ornitólogo Dorst, se desempeñó como titular de cátedra en el $\mathrm{Mu}$ seo de Historia Natural en París, donde abarcó el estudio de la sistemática, distribución comportamiento y biología de las aves y mamíferos. El libro narra acerca de las relaciones del hombre con la naturaleza y presenta una prodigiosa síntesis de todos los hechos ocurridos desde que el hombre aparece sobre la faz de la tierra hasta mediados del siglo XX. Desde el primer capítulo: el hombre preindustrial y su influencia sobre la naturaleza, seguido de los capítulos, el hombre en lucha con la naturaleza, el hombre en auxilio de la naturaleza, la explosión demográfica del siglo XX, a hierro y fuego: la destrucción de las tierras por el 
hombre, males y remedios perniciosos, la ordenación racional de la Tierra, hasta el último capítulo, hacia la reconciliación del hombre con la naturaleza; el rigor con el que han sido presentado los sucesos permite visualizar con claridad los acontecimientos secuenciados históricamente, los mismos que han sido enriquecidos con datos, citas y pasajes que le dan una dimensión y relevancia a la obra realmente impresionante.

Dorst señala que el problema del hambre no es el único que se agudiza día a día. Los factores políticos inspirados en un anticolonialismo desfasado también han contribuido a ello. Para el investigador la conservación de la naturaleza y la explotación de los recursos son problemas que existen desde que el hombre apareció sobre la faz de la tierra. La especie humana ha ejercido, más que ninguna otra especie, presión sobre los diversos tipos de hábitats, causando en un sin número de casos desequilibrios en contra de la naturaleza. En el contexto histórico de la Tierra, biológicamente la aparición del hombre causó un gran efecto en la transformación del mundo. Los cambios producidos en las poblaciones animales y vegetales, a lo largo de la historia, no fueron tan impactantes como la aparición del hombre en la Tierra. El hombre ha desarrollado muchas luchas para asegurar su existencia, especialmente desde el Paleolítico al Neolítico, obligado a recolectar y cazar como estrategia de sobrevivencia. Muy diferente al afán de lucro y al sadismo por la masacre que se manifestó especialmente en Europa entre los siglos VII al XVI, con la consiguiente desaparición de grandes extensiones de bosques y pérdida de especies valiosas, como el gran pingüino, el bisonte, el rinoceronte, el dinornis; el exterminio en África en 1880, de más de 60000 elefantes, y el registro de la salida de África francesa de 750000 pie- les de antílope, y que dos millones de antílopes enanos fueron abatidos en un solo año.

Con el paso del tiempo, el establecimiento de parques nacionales y otras áreas protegidas y los esfuerzos por conservar especies animales y de plantas han costado denodados esfuerzos. El progreso técnico y científico está cada día más lejos de los hombres del paleolítico que recogían los frutos caídos de los árboles en el suelo; esta situación es ahora solo propia de las comunidades indígenas en estado de aislamiento; tal como sucede en muchos casos en la Amazonía. Pero también, con algo de alarma, Dorst afirma que el hombre de manera imprudente ha hecho de aprendiz de brujo porque ha puesto en marcha una serie de procesos, que actualmente no es capaz de dominar y que atentan contra el equilibrio natural de la biósfera (Dorst, 1987).

\section{La primavera silenciosa}

Rachel Louise Carson nació el 27 de mayo de 1907, en un pequeño pueblo al sur de Pennsylvania, en los Estados Unidos de Norte América. Estudió en el colegio Springdale hasta el décimo curso y culminó la secundaria en el Instituto Parnassus de Pensilvania en 1925, en el primer lugar de su promoción. Continuó sus estudios superiores de Literatura Inglesa en un Colegio de Pensilvania para Mujeres; y en el tercer año, cambió a la carrera de biología. Posteriormente, continuó sus estudios de maestría en biología marina, en la Universidad Johns Hopkins, los que culmina en 1932, con la calificación cum laude.

Rachel Carson inició sus actividades profesionales en 1935, en el gobierno federal, primero en la Dirección de Pesca y después en el Servicio de Pesca y Vida Silvestre (Fish and Wildlife Service - US), que había sido creado por aquel entonces; en 1936 asume un nuevo 
cargo como bióloga marina, y se convierte en una de las primeras mujeres que llega a una posición de liderazgo dentro de la institución, donde escribió numerosas publicaciones para informar a las personas acerca de las variedades de pescado de importancia alimenticia, de las medidas para la conservación de los peces, restricciones sobre su población para evitar la sobreexplotación, épocas de veda, etc. De este modo, a través de sus publicaciones en la revista Progressive Fish-Culturist y folletos, animaba a la gente a consumir más pescado; lo que también le permitió ascender progresivamente a posiciones cada vez más importantes en el Servicio de Pesca y Vida Silvestre; institución de la cual se retiró en 1952, con el cargo de jefe de redacción de publicaciones.

Carson fue una reconocida investigadora, y una destacada escritora de temas científicos $\mathrm{y}$ ambientales, de una prosa liviana y clara y con una gran capacidad de comunicación. Ella era una persona convencida de que no había una línea divisoria entre la literatura y la ciencia. En esa perspectiva, educó a sus lectores a través de la pasión que puso en sus escritos; fue razón y sentimiento, dedicación, entrega, profundidad y convicción, a la vez. Una mujer íntegra comprometida con la ciencia y la naturaleza; el éxito económico solo era importante en la medida que le ofrecía posibilidades para dedicarse a escribir, sobre lo que más le interesaba: la vida silvestre, el tema ambiental.

Por aquel entonces, en 1944, algunos científicos venían advirtiendo sobre el riesgo que producía el uso del DDT, debido a que ocasionaba la muerte de los insectos dañinos y benéficos y como consecuencia de ello se alteraba el equilibrio en la naturaleza. Muchos fueron los artículos científicos y de divulgación, que mostraban una serie de hechos y descubrimientos, los que y se constituían en valiosos relatos sobre las consecuencias perniciosas del uso de los pesticidas en el largo plazo. Poco tiempo después, en la década del cincuenta, se encuentran evidencias de la acción perniciosa del DDT en la cadena alimenticia, de sus niveles de concentración y acumulación en los tejidos grasos de los organismos; sin embargo, lo más lamentable es que dicha información era conocida sólo por un reducido grupo de científicos.

Es así como se llega al año 1962, cuando Rachel Carson escribió el famoso y trascendental libro la Primavera silenciosa, donde relataba una serie de situaciones que afectaban a la vida silvestre en la naturaleza, en un escenario caracterizado por un futuro silencioso: $\sin$ el alegre canto de los pájaros al amanecer, el colorido vuelo de las mariposas en las mañanas soleadas y la oscura degradación de la naturaleza producida por la contaminación ambiental. En aquella época, había una enorme discusión sobre los beneficios y consecuencias del uso del DDT, un pesticida intensamente utilizado para controlar las plagas en la agricultura, que, entre otros, causaba grandes estragos sobre la población del águila calva, el ave emblemática de los Estados Unidos.

Rachel Carson estaba convencida de que el tema era sumamente polémico y que tocaba fibras sensibles de muchas instituciones públicas y privadas, sobre todo de aquellas dedicadas a la agroindustria. Por ello, precisamente, fue injustamente acusada de alarmista, maltratada sin misericordia por cierto sector de la prensa ligada a poderosos intereses económicos poderosos y demandada ante la justicia. Sin embargo, lo más importante fue la visión que se extendió por todo el mundo acerca del impacto negativo de los pesticidas, que ejerció especialmente el DDT, sobre la destrucción de 
la naturaleza. La Primavera silenciosa fue la primera y más impactante declaración pública de que los plaguicidas estaban causando enorme daño al ambiente y a los organismos vivos. Escribir dicha obra fue todo un desafío, un extraordinario reto hasta ahora no imaginado, para una época donde, el poder económico y la industria, no iban a permitir ese tipo de románticos idealismos. Más aún, provocó una gran controversia sobre el uso pernicioso de los pesticidas químicos porque alertó al público de los peligros que implicaban la aplicación indiscriminada sobre los cultivos agrícolas. A través de documentos cuidadosamente seleccionados y analizados, se determinó la enorme cantidad de efectos dañinos que los pesticidas ocasionaban; pero todavía más, se demostró cómo los pesticidas causaban más daño al ambiente que las plagas que se buscaba erradicar. $Y$ es que era escaso el conocimiento acerca de los efectos de los pesticidas en el largo plazo sobre el ambiente, la vida silvestre y los seres humanos. Pero también demostró la vigorosa interrelación existente entre las plantas, los animales y los seres humanos y la comprensión de que el hombre como especie, es uno más, forma parte de la naturaleza, al igual que el resto de seres vivos y que dependemos unos de otros, para garantizar la sobrevivencia de nosotros mismos en la Tierra (Cuenca, 2006).

La Primavera silenciosa ha sido considerado uno de los 100 libros de ciencia más importantes del siglo XX; una referencia de lectura obligada de los ambientalistas a nivel mundial, que resaltó el peligro del uso de los plaguicidas para la naturaleza y todas las comunidades vivientes, lo que provocó que los agricultores, industriales, científicos, ambientalistas y hasta desde el propio gobierno, levantaran su voz de protesta. Poco tiempo después de su publicación, diferentes personas e instituciones se unieron a esta gran cruzada contra los pesticidas venenosos y se formaron organizaciones dedicadas a la defensa de un ambiente sano y limpio, así como también con el tiempo, se implementó legislación específicamente relacionada con los temas ambientales. Los años 60, marcan un hito en la historia, pues son reconocidos como el nacimiento del movimiento ambientalista moderno, el cual tuvo un tremendo impacto, a tal extremo que el Congreso de los Estados Unidos aprueba en 1970, la creación de la EPA (Environmental Protection Agency), la primera agencia gubernamental dedicada exclusivamente a la protección del ambiente.

Antes de la publicación de la Primavera silenciosa, los residuos de los procesos químicos eran liberados indiscriminadamente al aire, agua y suelo; sin tener en cuenta el límite de la capacidad biótica del planeta Tierra, para los procesos de trasformación de la materia orgánica producida por parte de las actividades generadas por los seres humanos. Después de la Primavera silenciosa, la gente se da cuenta, con asombro, que los productos químicos liberados al ambiente no solo generaban problemas por su toxicidad, sino también que tenían una gran capacidad de persistir en los organismos a través de la acumulación en los tejidos grasos, hasta llegar a niveles incontrolables.

La Primavera silenciosa fue el último libro que Carson escribió hasta antes de su muerte, el 14 de abril de 1964. Su muerte prematura le impidió ver el impacto que su obra tuvo en la gente, en la sociedad toda, en el mundo académico, en la ciencia. La publicación de la Primavera silenciosa en 1962 y otros escritos suyos, como artículos y libros de divulgación científica contribuyeron a la puesta en marcha de la moderna conciencia ambiental; de la actitud que debe adoptar la ciudadanía ante 
hechos que atentan contra los animales y las plantas que viven en armonía en la naturaleza, para así garantizar una vida sana, un futuro digno y responsable para la especie humana.

\section{Nuestro futuro robado}

El libro Nuestro futuro robado fue escrito por Theo Colborn, Dianne Dumanoski y John Peterson Myers en 1996; en sus páginas se dan a conocer una serie de sorprendentes hallazgos y transmite un mensaje que trasciende los conocimientos tradicionales sobre las sustancias químicas sintéticas, su seguridad y la manera de prevenir sus peligros; así como se difundió información desconocida para la gran mayoría de la gente, y por si esto fuera poco, estos contaminantes llegaron a convertirse en parte integrante de nuestra economía industrial. Naturalmente que la única forma de pasar por encima de la barrera de los intereses privados, es que duda queda, la explosiva diseminación del conocimiento.

Si el libro Primavera silenciosa escrito por Rachel Carson, informó por primera vez sobre la peligrosidad de los contaminantes en su tiempo y previno sobre las repercusiones de los plaguicidas y otras sustancias químicas sintéticas; Nuestro futuro robado pone en evidencia el riesgo del uso de productos químicos, que ocasionan el cáncer y otras enfermedades; cómo es el caso de sustancias químicas artificiales que suplantan a las hormonas naturales trastornando los procesos normales de desarrollo y reproducción en el hombre y los animales, con lo que se está poniendo en serio peligro el futuro de la humanidad. En el libro no solo se dan cuenta de las consecuencias que ha ocasionado el uso de algunos productos químicos, sino que además tuvo la gran virtud de que se reunieron evidencias científicas, que Carson esperó durante mucho tiempo y que desafor- tunadamente no pudo contar con ellas cuando escribió su libro la Primavera silenciosa.

Es por ello que como resultado de los descubrimientos efectuados en investigaciones sobre los disruptores hormonales, se pusieron en evidencia una serie de hechos que llamaron poderosamente la atención. Al respecto, se presenta una serie de datos históricos, desde 1952, cuando muchas especies animales, localizadas en diferentes áreas del planeta, presentaban alteraciones en su sistema hormonal debido a la exposición a sustancias químicas artificiales, los cuales sugieren que los contaminantes pudieron haber interferido con el control hormonal del desarrollo en las especies de animales y en las personas. Estas alteraciones incluyen la pérdida de la capacidad reproductora, mortandades masivas, deformaciones en órganos reproductores, comportamientos sexuales anómalos y disminución del sistema inmunológico de las especies afectadas por diferentes sustancias químicas; que alteran el desarrollo y el funcionamiento normal de los órganos sexuales; la aparición de peces hermafroditas o machos afeminados en ríos y efluentes de plantas depuradoras en diferentes países del mundo; la disminución, en las últimas décadas, en el hombre de casi un $50 \%$ de la cantidad de espermatozoides; y el cáncer relacionado con las hormonas que ha ido en aumento en las mujeres.

De igual manera, es interesante porque se mencionan algunos casos muy ilustrativos como el de las nutrias que estuvieron a punto de desaparecer de los ríos en Inglaterra (1950); el águila calva en Florida (1952), en la cual se determinó la pérdida del instinto natural de apareamiento y cría, encontrándose que un $80 \%$ de las águilas eran estériles; en los años 60, las granjas cercanas al Lago Mi- 
chigan (Grandes Lagos, USA), las hembras de visón habían disminuido su nivel de fertilidad y perdían sus crías, tiempo después se detectó que esto se debía a la presencia de bifenilos policlorados, que son compuestos pertenecientes a la llamada docena sucia; en los años 70 , se detectó que las hembras de la gaviota occidental del sur de California (USA) formaban pareja con otras hembras; en 1970, el $80 \%$ de los polluelos de gaviotas argénteas del Lago Ontario moría antes de salir del huevo y algunos tenían deformidades parecidas a las de los pollos tratados con dioxinas en experimentos de laboratorio. A finales de los años 80, los caimanes del Lago Apopka de Florida (USA) nacían con el pene de tamaño muy pequeño, lo que impedía su apareamiento con lo que descendió su eficacia reproductiva; sólo el $18 \%$ de los huevos eran viables, la mitad de las crías morían antes de los 10 días, el 60\% de los machos presentaban penes anormalmente pequeños, con caracteres feminizados y las hembras adolescentes presentaban deformaciones en los ovarios y su nivel de estrógenos en sangre era el doble de lo normal; todo ello como consecuencia de que se había vertido insecticidas dicofol y DDT, desde una fábrica cercana hacia el lago. Las focas en el norte de Europa (1988) sufrían los efectos de la baja fertilidad; y el delfín en el Mediterráneo (1990) afrontaba una mortandad masiva ocasionada por una infección viral; y al analizar los animales muertos se encuentran concentraciones de PCBs, de hasta tres veces superiores con respecto a los animales sanos. En Inglaterra (1990) se encontró la feminización de peces que vivían a la salida del vertedero de aguas residuales, anormalidades que no se encontraban en peces que vivían aguas abajo; por lo que se sospechó de unas sustancias químicas procedentes de la degradación de detergentes y plásticos, los alquilfenoles.
Todo ello coincide con que, a partir de 1950, tanto el auge económico, el desarrollo de la investigación científica y la innovación tecnológica generaron nuevos productos industriales y paralelamente aparecieron estos problemas en diferentes partes del mundo, a los cuales no se les encontró respuesta. Se tenía nueva información sobre órganos sexuales defectuosos y anomalías de conducta, pérdida de la fecundidad, alta mortalidad juvenil y lo más desconcertante la desaparición inexplicable de poblaciones animales enteras.

Por ello Nuestro futuro robado hace una contribución muy importante, al tratar de interpretar estos problemas que afectan a la especie humana a causa de los disruptores endócrinos: anomalías genitales en los niños, cáncer de testículo y endometriosis, crecimiento de la glándula prostática, problemas de reproducción en las mujeres, abortos, embarazos ectópicos, cáncer de mama con tasas en crecimiento, etc. Es así como ha llamado mucho la atención una serie de problemas reproductivos que han afectado a los seres humanos. La cantidad media de espermatozoides en los hombres había disminuido en un 45\% entre 1940 y 1990, al tiempo que el volumen de semen eyaculado había disminuido un 25 por ciento, mientras que la cantidad de espermas defectuosos ha aumentado en cada eyaculación. Aunque no se sabe a ciencia cierta las cantidades de las sustancias químicas disruptoras que constituyen un peligro para el ser humano, estas podrían ser muy pequeñas si la exposición se presenta durante la gestación. Los estudios recientes han demostrado que la exposición de dosis ínfimas de las dioxinas resulta peligrosa; cantidades insignificantes como una décima parte por billón de estrógeno libre pueden alterar el curso del desarrollo en el útero. Las investigaciones científicas han compro- 
bado que actualmente todos los humanos, sin excepción, tienen en el cuerpo 500 sustancias químicas, que no existían en las personas hace cincuenta años. Pero a la vez, una gran cantidad de sustancias químicas, amenazan la fecundidad, la inteligencia y la supervivencia del ser humano. Hoy en día se encuentran en el mercado unas 100000 sustancias químicas sintéticas; pero cada año se introducen 1000 nuevas sustancias, la mayoría de ellas sin siquiera haber pasado por un adecuado control de calidad. Las investigaciones han permitido identificar a 51 productos químicos que alteran el sistema hormonal, sin embargo, no se conocen los efectos fisiológicos o anatómicos de la mayoría de ellos. Pero lo más alarmante y que preocupa a la comunidad científica y a la sociedad es que los fabricantes, al amparo de la ley, niegan el acceso a la información sobre la composición de sus productos (Colborn et al, 2001).

\section{Los impactos de Antes que la naturaleza} muera, La primavera silenciosa y Nuestro futuro robado

La publicación de Antes que la naturaleza muera, la Primavera silenciosa y Nuestro futuro robado, generaron una serie de impactos en la sociedad civil y en los gobiernos y a la vez diferentes tipos de corrientes de opinión, que duda queda. Así tenemos que en 1968 se reunieron en la ciudad de Roma, científicos y políticos provenientes de 30 países, a fin de discutir acerca de los cambios que se estaban produciendo en el planeta a consecuencia de las actividades humanas y con una gran preocupación por mejorar el futuro del mundo en el largo plazo. Producto de esta reunión se conformó el "Club de Roma", el cual encargó al Massachusetts Institute of Technology (MIT) para que un grupo de 17 científicos a nivel mundial, liderados por Donella Mea- dows, biofísica y científica ambiental, especializada en dinámica de sistemas, procediera en 1972, a la elaboración de la publicación "Los límites del crecimiento". Este informe que se preparó antes de la primera crisis del petróleo, dio el impulso inicial para la formación de movimientos ecológicos y ambientalistas en el mundo (Cuéllar et al. 2010).

Un hito fundamental en la historia del ambientalismo fue la Conferencia de las Naciones Unidas sobre Ambiente Humano que se dio lugar en Estocolmo, Suecia en el año 1972. El título de la conferencia "Sólo una Tierra" ha sido el símbolo de un cambio en la comprensión humana que se convertiría en un elemento fundamental en el desarrollo de la ética y en el planteamiento de las políticas ambientales. Lo más significativo de esta conferencia fue el hecho de que se sentaron las bases de lo que más tarde se reconocería como sustentabilidad (Calvente, 2007).

Pocos años después, la primera Conferencia Intergubernamental sobre Educación Ambiental celebrada en Tbilisi, Georgia, en octubre de 1977, organizada por la Unesco en colaboración con el Programa de la Naciones Unidas para el Medio Ambiente (PNUMA), como una prolongación de la Conferencia de las Naciones Unidas sobre el Medio Humano (Estocolmo, junio de 1972), cuyas implicancias en aspectos referidos a la educación, se reflejaron en el capítulo titulado "El hombre y su medio ambiente" del Plan de la Unesco (1977-1982), aprobado en la XIX Conferencia General en Nairobi en 1976. Durante esta conferencia se trataron temas como la protección del ambiente, la búsqueda de relaciones comunes entre ambiente y economía. Uno de los resultados de esta conferencia fue la creación del Programa Ambiental de las Naciones Unidas (UNEP). 
Todo ello generó un nuevo concepto del desarrollo, en el cual se involucra la satisfacción de las necesidades y requerimientos de las personas, el pluralismo de las sociedades y la búsqueda del equilibrio entre el hombre y la biosfera. Es aquí donde aparece la opción holística "como una perspectiva en la cual se demuestra que todo está íntimamente ligado con las interrelaciones de los organismos vivos en nuestro planeta" (Gil \& Vilches. 2004).

En el año 1983 las Naciones Unidas crearon la Comisión Mundial de Ambiente y Desarrollo (WCED), presidida por Gro Harlem Brundtland, primer ministro de Noruega y uno de los resultados más importantes fue la de identificar iniciativas desde tres enfoques: económico, ambiental y social. En el informe Brundtland, "Nuestro futuro común", publicado en 1987, quedó definido el concepto de desarrollo sustentable, que fue aceptado por el Programa Mundial de Medio Ambiente y Desarrollo de Naciones Unidas (Reyes-Sánchez, 2012). Posteriormente, en el año 1992 se celebró la Cumbre de Río o de la Tierra en Río de Janeiro, Brasil del 3 al 14 de junio de 1992, con la asistencia de 120 países y 400 representantes de organizaciones no gubernamentales $(\mathrm{ONG})$ y donde se consolida el accionar de las Naciones Unidas con respecto a los conceptos de ambiente y desarrollo sustentable. Se acordaron 27 principios relacionados con la sustentabilidad que se consolidan en la Agenda $21 \mathrm{y}$ donde se adopta un programa de acción para el siglo XXI, que presenta 2500 recomendaciones relativas a la salud, vivienda, contaminación del aire, la gestión de los mares, biodiversidad, bosques y montañas, la desertificación, recursos hídricos y saneamiento, agricultura y gestión de residuos. La Declaración de Río reafirma con vigor el concepto de desarrollo sostenible.
Diez años después de la cumbre de Río de Janeiro en 1992, se realizó la de Johannesburgo en 2002 y seguidamente se celebró del 20 al 22 de junio de 2012 en Río de Janeiro, Brasil la Cumbre de la Tierra, llamada oficialmente Conferencia de Naciones Unidas sobre Desarrollo Sustentable, que fue un nuevo intento de Naciones Unidas para avanzar en el compromiso de los Estados y la comunidad internacional en asumir responsablemente los grandes cambios ambientales del siglo XXI y "sentar las bases de un mundo de prosperidad, paz y sustentabilidad" (Pulido \& Olivera, 2018).

Asimismo, cada 26 de noviembre se conmemora el Día Mundial Contra el Uso Indiscriminado de Agroquímicos, establecida por 400 organizaciones en 60 países, recordando a los miles de personas fallecidas, a consecuencia de los accidentes de Seveso en Italia en 1976, Bophal en la India en 1984, y Vera Cruz en México en 1991. Esta fecha fue adoptada para tomar conciencia por parte de la población mundial, sobre la grave crisis ambiental generada por el uso de los agroquímicos.

A nivel regional se ha concluido el proceso para lograr la próxima entrada en vigor del Acuerdo Regional sobre el Acceso a la Información, la Participación Pública y el Acceso a la Justicia en Asuntos Ambientales en América Latina y el Caribe, más conocido como Acuerdo de Escazú; que es un tratado internacional que garantiza el ejercicio de los derechos de acceso a información, participación ciudadana y justicia ambiental, así como el fortalecimiento de la protección de los defensores ambientales en Latinoamérica y El Caribe. Aunque el acuerdo fue adoptado en Escazú (Costa Rica), el 4 de marzo de 2018, y tuvo 24 países firmantes, debía tener como mínimo la ratificación de 11 países para en- 
trar en vigor. La entrada en vigor del Acuerdo de Escazú tendrá como escenario el Día Internacional de la Tierra, 22 de abril de 2021.

\section{A manera de colofón}

Desde la aparición del hombre en la Tierra, la historia señala su papel contradictorio. Por un lado, el hombre ha devastado especies y destruidos bosques y otros tipos de hábitats, pero también ha hecho esfuerzos por establecer áreas protegidas, conservar la biodiversidad y llegar a la firma de acuerdos internacionales para establecer mecanismos entre las naciones para proteger la naturaleza. Después de la segunda guerra mundial las condiciones sanitarias mejoraron en los países y las causas de muerte se fueron desplazando de las enfermedades infecciosas a las enfermedades circulatorias, y al cáncer; y por tanto un producto era considerado tóxico cuando provocaba cáncer o malformaciones congénitas en las personas. Muchos compuestos químicos pueden provocar cáncer, pero también alteraciones hormonales que hacen disminuir la fertilidad de las personas, que afectan el crecimiento y desarrollo o el nivel de inteligencia, que incentiven la hiperactividad o disminuyan la capacidad de concentración. Los compuestos químicos como el bifenil polychlorinados (PCBs) un organoclorados que contamina el agua y los sedimentos, es consumido por microorganismos $\mathrm{y}$ estos a su vez por pequeños animales, que son devorados por organismos más grandes como las aves, los mamíferos y los humanos, y así se va de eslabón en eslabón, construyendo el tejido de la cadena trófica. Estos químicos se acumulan en el ser humano que está en el lugar más alto de la cadena alimenticia y en la acumulación exponencial de PCBs. La toxicidad de compuestos químicos como los PCBs es más nocivo en la descendencia porque atraviesan la placenta y pasan de la madre al feto en las primeras etapas del ser vivo que en los adultos. Y los estrógenos tienen efectos diferentes según el sexo, y la edad, por lo que el momento y la etapa de desarrollo y el sexo son objeto de diferentes grados de toxicidad. Tal es el caso de los estrógenos sintéticos como el dietilestilbestrol (DES) que fueron vendidos en las décadas de los cuarenta y cincuenta, del siglo pasado, para obtener una descendencia robusta y suprimir la leche materna después del parto, así como en el tratamiento del cáncer de próstata y otras dolencias. En los años 70 se empezaron a reconocer los efectos que el DES producía en los niños que nacían mutilados sin piernas, brazos o sin dedos; a esto se le llamó el caso de la talidomida; que fue prohibida en Estados Unidos de Norte América en 1976. Lo que confirma que el organismo no tiene la suficiente capacidad de eliminar estos compuestos sintéticos, como lo hace, con los estrógenos naturales.

La bióloga Rachel Carson presagió un planeta silencioso y dolorosamente sombrío, por el uso indiscriminado de productos químicos, que afectan al ambiente físico, a los microrganismos, las plantas, los animales y el hombre; y que Colborn, Dumanoski y Myers sostienen que se revierte hacia el ser humano y es causa fundamental del origen de varias enfermedades, con lo que se demuestra la estrecha relación entre el ambiente y la salud. Por lo tanto, la realidad problemática ambiental es el resultado del desarrollo de las diferentes actividades humanas. Así tenemos que la contaminación ambiental, la pérdida de la biodiversidad, la erosión de los suelos, el agotamiento de la capa de ozono, el calentamiento global, la deforestación de los bosques y la pobreza son las consecuencias de la actitud negligente del hombre en su relación con el planeta. Por ello, hoy en día el análisis de los asuntos bioéticos pone en relieve la importancia de los problemas sociales y ecológicos, por lo que, 
se reconoce la necesidad de una reflexión de carácter moral. Visto desde esa perspectiva, la bioética y la educación ambiental, forman parte del mismo discurso que relaciona lo científico y lo humano. La ética ambiental tiene sentido en la medida que las personas y las sociedades, adoptan un comportamiento de respeto hacia los otros seres vivos, así como a la conservación del planeta. No obstante, las personas no reconocen en la naturaleza derechos que se deban respetar; y los derechos humanos sobre la naturaleza se ha convertido en aspectos económicos, políticos, legales y quizá morales.

Rachel Carson, Jean Dorst, Colborn, Dumanoski y Myers y una gran cantidad de científicos no solo dieron la voz de alarma, sino que asumieron una posición crítica y de liderazgo en momentos trascendentales. Por ello en las actuales circunstancias es nuestro deber como ciudadanos de bien construir una sociedad justa, equitativa y equilibrada donde el ambiente sano que genera prosperidad y visión de futuro sea una prioridad tanto como la estabilidad política, económica y social de un país.

\section{REFERENCIAS BIBLIOGRÁFICAS}

Calvente, A. M. (2007). El Concepto Moderno de Sustentabilidad. UAIS. Sustentabilidad. http://sustentabilidad.uai.edu.ar/pdf/cs/UAISCS-200-001\%20-\%20Planetarizacion.pdf

Carson, R. (2010). Primavera Silenciosa. Editorial Crítica.

Colborn, T., Myers, J. P., \& Dumanoski, D. (2001). Nuestro Futuro Robado ¿Amenazan las sustancias químicas sintéticas nuestra fertilidad, inteligencia y supervivencia? Editorial Ecoespaña.
Cuéllar, L., Serra S., Collado A., \& Reyes, R. (2010). La bioética desde la perspectiva de la salud ambiental: su expresión en Cuba. Revista Cubana de Higiene y Epidemiología, 48(3),321334. http://scielo.sld.cu/scielo.php?script=sci_ arttext\&pid=S1561-30032010000300011

Cuenca, R. E. (2006). ¿La bioética en la educación ambiental? Colombia Médica, 37(4), 299-307. https://www.redalyc.org/ pdf/283/28337408.pdf

Dorst, J. (1987). Antes que la naturaleza mиera. Ed. Omega. $2^{\text {a }}$ edición. Barcelona.

Gil, D. \& Vilches, A. (2004). La contribución de la ciencia a la cultura ciudadana. Cultura y Educación, 16(3), 259-272.

Pulido, V. \& Olivera, E. (2018). Aportes pedagógicos a la educación ambiental: una perspectiva teórica. Revista de Investigaciones Altoandinas, 20(3), 333-346. https://dx.doi. org/10.18271/ria.2018.397

Reyes-Sánchez, L. B. (2012). Aporte de la química verde a la construcción de una ciencia socialmente responsable. Educación química, 23(2), 222-229. https://www.sciencedirect. com/science/article/pii/S0187893X17301131 